\title{
Effet du déficit hydrique induit par l'agar-agar sur la croissance des vitroplants de deux morphotypes de voandzou (Vigna subterranea L. Verdc.)
}

\author{
Yaya TOURE $^{1 *}$, Tchoa KONE ${ }^{2}$, Lacina Fanlégué COULIBALY ${ }^{3}$, Nakpalo SILUÉ ${ }^{4}$ et \\ Mongomaké KONE ${ }^{2}$
}

${ }^{1}$ Université de Man, UFR Ingénierie Agronomique, Forestière et Environnementale, Man, Côte d'Ivoire.

${ }^{2}$ Université Nangui Abrogoua, UFR Sciences de la Nature, Abidjan, Côte d'Ivoire.

${ }^{3}$ Université Péléforo Gon Coulibaly, UFR des Sciences Biologiques, Korhogo, Côte d'Ivoire.

${ }^{4}$ Silué Nakpalo, Université Félix Houphouët-Boigny, UFR Biosciences, Abidjan, Côte d'Ivoire.

*Auteur correspondant ; E-mail : toureyaya12@yahoo.fr

\begin{tabular}{ccc}
\hline Received: 06-07-2021 & Accepted: 23-11-2021 & Published: 31-12-2021 \\
\hline
\end{tabular}

\section{RESUME}

Le voandzou (Vigna subterranea L.) est l'une des légumineuses à graines tropicales généralement cultivé dans les régions à faible pluviométrie. Cette étude visait à déterminer le comportement des morphotypes Ci2 et $\mathrm{Ci} 4$ de voandzou en conditions de déficit hydrique. Les vitroplants issus du développement des axes embryonnaires des graines et âgés de dix jours, ont été transférés sur le milieu de base de Murashige et Skoog (1/2MS0) solidifié avec différentes concentrations d'agar-agar (6 g/l, 12 g/l, 18 g/l, 24 g/l, 30 g/l, 36 g/l) pour induire le déficit hydrique. Après 30 jours d'incubation, l'évaluation des vitroplants a montré que l'effet du déficit hydrique sur leur croissance a varié selon l'organe et le morphotype utilisé. Le système racinaire des plantules a été plus affecté par le déficit hydrique comparativement au système aérien. Les taux de réduction de la hauteur des plantules, du nombre de feuilles, de la biomasse fraîche aérienne et de la biomasse sèche racinaire ont respectivement été de 39,46\%, 16,66\%, 72,88\%, 29,39\%, 32,50\%, 52,25\% pour le morphotype Ci2 et de $45,94 \%, 39,40 \%, 72,15 \%, 53,80 \%, 39,18 \%$ 67,93\% pour le morphotype Ci4. La réduction de la croissance consécutivement au déficit hydrique induit par l'agar-agar a été plus importante avec le morphotype Ci4. Aussi, le morphotype Ci2, dont la réduction de la croissance a été moins affectée est considéré comme étant le plus tolérant au déficit hydrique.

(C) 2021 International Formulae Group. All rights reserved.

Mots clés : Vigna subterranea, morphotype, agar-agar, vitroplants, déficit hydrique, tolérant.

\section{Effect of water deficit induced by agar-agar on the vitroplants growth of two landraces of voandzeia (Vigna subterranea $\mathrm{L}$. Verdc.)}

\begin{abstract}
ABSRACT
Voandzeia (Vigna subterranea L.) is one of the most common tropical legumes grown in low rainfall areas. The objective of this study were to determine the behavior of two landraces (Ci2 and Ci4) under water deficit conditions. Vitroplants from the development of the embryonic axes of seed, aged ten days, were transferred to the base medium of Murashige and Skoog ( $\left.1 \frac{1}{2} \mathrm{MS} 0\right)$ solidified with different concentrations of agaragar $(6 \mathrm{~g} / \mathrm{l}, 12 \mathrm{~g} / \mathrm{l}, 18 \mathrm{~g} / \mathrm{l}, 24 \mathrm{~g} / \mathrm{l}, 30 \mathrm{~g} / \mathrm{l}, 36 \mathrm{~g} / \mathrm{l})$ to induce water deficit. After 30 days of incubation, vitroplant evaluation showed that the effect of water deficit on growth varied depending on organ and landrace used.
\end{abstract}


Seedling root systems were more affected by water deficit than the aerial system. The reduction rates of seedling height, leaf number, aerial fresh biomass, and root dry biomass were respectively $39.46 \%, 16.66 \%, 72.88 \%$, $29.39 \%, 32.50 \%, 52.25 \%$ for landrace $\mathrm{Ci} 2$, and $45.94 \%, 39.40 \%, 72.15 \%, 53.80 \%, 39.18 \% 67.93 \%$ for landrace $\mathrm{Ci} 4$. The reduction in growth following agar-agar induced water deficit was greater with morphotype Ci4. Also, landrace $\mathrm{Ci} 2$, whose reduction in growth has been less affected, is considered to be the most water deficit tolerant. (C) 2021 International Formulae Group. All rights reserved.

Keywords: Vigna subterranea, landrace, agar-agar, vitroplants, water deficit, tolerant.

\section{INTRODUCTION}

Dans les zones semi-arides tropicales, les pluies sont sporadiques et une grande partie de ces pluies est souvent perdue par le ruissellement et l'évaporation. Seule une faible proportion est disponible pour les plantes. Afin de s'adapter à ce déficit, la majorité des plantes des régions semi-arides ont développé des mécanismes pour assurer leur survie et leur reproduction. Ces mécanismes d'adaptation varient considérablement d'une espèce à une autre et même au sein d'une même espèce (Lavorel, 2013).

Le voandzou (Vigna subterranea L.) est majoritairement cultivé par les femmes en Afrique (Touré et al., 2013; Ouoba et al., 2016) pour ses graines riches en protéine. C'est l'une des légumineuses à graines tropicales considérée comme tolérante à la sécheresse (Basu et al., 2007). Cependant, très peu de travaux ont été rapportés dans la littérature pour soutenir cette capacité d'adaptation à la sécheresse ainsi que les mécanismes développés par la plante. A partir des essais réalisés en conditions de champ, Mwale et al. (2007) ont suggéré que la tolérance au déficit hydrique chez le voandzou se fait par ajustement osmotique, par réduction de la surface foliaire et par une faible perte d'eau par les stomates. Ces deux caractères sont considérés comme étant des critères liés à la tolérance en eau. Toutefois, les principaux caractères significatifs de la tolérance à la sécheresse (la croissance de la racine, l'utilisation de l'eau et la productivité) n'ont pas encore été suffisamment étudiés chez le voandzou.

Les premières stratégies développées pour cribler les espèces végétales quant à leur tolérance à la sécheresse ont été basées sur l'évaluation de la biomasse sèche et de la surface foliaire après irrigation et suspension de l'irrigation dans les conditions de champ. Dans ces conditions, l'effet du déficit hydrique n'est généralement pas indépendant, mais résulte de l'interaction d'un complexe de facteurs environnementaux. Pour surmonter ces difficultés, il a été développé une technique in vitro utilisant l'agar-agar pour induire le déficit hydrique. L'objectif de ce travail était de mettre en évidence les potentialités d'adaptation à la sécheresse de deux morphotypes de voandzou en conditions de culture in vitro.

\section{MATERIEL ET METHODES}

Le matériel végétal utilisé était constitué des graines de deux morphotypes de voandzou (Figure 1) originaire de la zone savanicole de la Côte d'Ivoire. Ces morphotypes ont en commun la durée du cycle (4 mois) mais diffèrent par la couleur du tégument et la forme des graines (Touré et al., 2012).

\section{Désinfection en surface des graines}

Sous une hotte à flux laminaire, les graines ont subi une désinfection par trempage dans l'alcool $70 \%(\mathrm{v} / \mathrm{v})$ pendant une minute, puis une immersion dans une solution d'hypochlorite de calcium $7 \%(\mathrm{~m} / \mathrm{v})$ pendant $30 \mathrm{mn}$. Après trois rinçages à l'eau distillée stérile, les graines ont été incubées dans l'eau distillée stérile et placées dans la salle de culture à l'obscurité pendant $48 \mathrm{~h}$ pour leur imbibition.

\section{Obtention des plantules in vitro et transfert sur les milieux de croissance}

L'axe embryonnaire (Figure 2A) des graines a été prélevé puis placé sur un milieu (Figure 2B) constitué des sels MS (Murashige et Skoog, 1962) réduite de moitié ( $1 \frac{1}{2}$ MS0). A 
ce milieu, ont été ajoutés $30 \mathrm{~g}$ de saccharose et $0,6 \mathrm{~g}$ d'agar.

Après dix jours d'incubation in vitro, les plantules homogènes (Figure 2C) de 2,5 $\mathrm{cm}$ de hauteur et dont la racine principale mesure 2,2 $\mathrm{cm}$, ont été transférées sur six milieux de croissance. Ces milieux ont été préparés de la même manière que le précédant milieu, mais différaient par leur concentration en agar (6 g/l, $12 \mathrm{~g} / \mathrm{l}, 18 \mathrm{~g} / \mathrm{l}, 24 \mathrm{~g} / \mathrm{l}, 30 \mathrm{~g} / \mathrm{l}$ et $36 \mathrm{~g} / \mathrm{l})$. Ces six milieux traduisent les différents niveaux de déficit hydrique. Le $\mathrm{pH}$ des milieux a été ajusté à 5,8 avec du $\mathrm{NaOH}(1 \mathrm{~N})$ ou du $\mathrm{HCl}(1 \mathrm{~N})$. Les cultures ont été transférées dans une salle de culture à une photopériode de $12 \mathrm{~h}$ et une température de $25 \pm 2{ }^{\circ} \mathrm{C}$. L'hygrométrie a été de $70 \%$ et l'intensité lumineuse de 2000 lux.

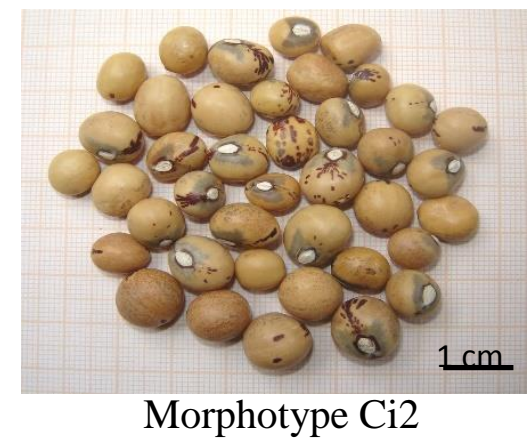

Les tubes ont été disposés dans la salle de culture suivant un dispositif complètement randomisé avec trois répétitions.

Après 30 jours d'incubation, les paramètres morphologiques et physiologiques évalués sur les plantules sont consignés dans le Tableau 1.

\section{Analyse statistiques des données}

L'analyse de variance à deux facteurs a été effectuée à l'aide du logiciel STATISTICA 6. Le premier était le facteur « morphotype » avec deux niveaux ( $\mathrm{Ci} 2$ et $\mathrm{Ci} 4)$ et le second était le facteur «concentration d'agar » avec six niveaux (6-36 g/l). Le test de Newman-keuls a servi à la comparaison de moyennes lorsque le $F$ de l'analyse de variance était significatif au seuil de 5\%.

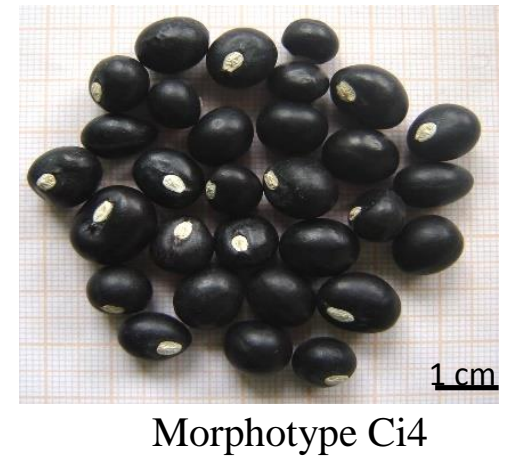

Figure 1: Graines des morphotypes Ci2 et Ci4 de voandzou.

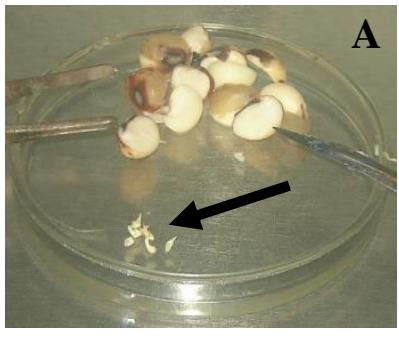

Embryons (flèche) débarrassés de ses cotylédons

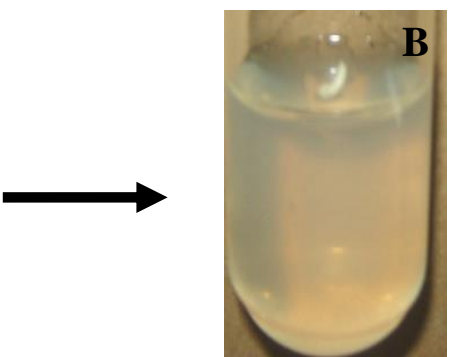

Embryon placé sur le milieu de germination

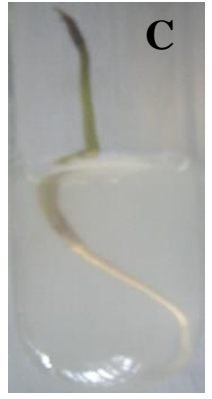

Plantules de 10 jours obtenues sur le milieu contrôle (6 g/l d'agar)

Figure 2: Obtention in vitro des plantules de voandzou. 
Tableau 1: Paramètres morphologique et physiologique évalués sur les plantules.

\begin{tabular}{|c|c|c|}
\hline Paramètres & Codes & Méthode de mesure \\
\hline $\begin{array}{l}\text { Hauteur de la plante }(\mathrm{cm}) \\
\text { Longueur de la racine principale }(\mathrm{cm})\end{array}$ & $\begin{array}{l}\text { Hpl } \\
\text { Lrp }\end{array}$ & Mesurées à l'aide d'une règle graduée \\
\hline $\begin{array}{l}\text { Nombre de feuilles } \\
\text { Nombre de racines secondaires }\end{array}$ & $\begin{array}{l}\mathrm{Nbf} \\
\mathrm{Nrs}\end{array}$ & Déterminés par comptage directe \\
\hline Surface foliaire $\left(\mathrm{cm}^{2}\right)$ & $\mathrm{Sf}$ & Calculée par la formule de Boudersa (1998) \\
\hline Contenu relatif en eau $(\%)$ & CRE & Calculé selon la méthode de Jensen et al. (2000) \\
\hline $\begin{array}{l}\text { Biomasse fraiche aérienne }(\mathrm{mg}) \\
\text { Biomasse fraiche racinaire }(\mathrm{mg})\end{array}$ & $\begin{array}{l}\mathrm{Bfa} \\
\mathrm{Bfr}\end{array}$ & Déterminées à l'aide d'une balance électronique \\
\hline $\begin{array}{l}\text { Biomasse sèche aérienne }(\mathrm{mg}) \\
\text { Biomasse sèche racinaire }(\mathrm{mg})\end{array}$ & $\begin{array}{l}\text { Bsa } \\
\text { Bsr }\end{array}$ & $\begin{array}{c}\text { Après séchage du matériel à l'étuve à } 70^{\circ} \mathrm{C} \text { pendant } \\
24 \mathrm{~h} \text {, prise de la masse à l'aide d'une balance } \\
\text { électronique }\end{array}$ \\
\hline $\begin{array}{l}\text { Ratio hauteur de la plante, longueur de } \\
\text { la racine }\end{array}$ & Rhl & $\begin{array}{l}\text { Quotient de la hauteur de la plante sur la longueur } \\
\text { de la racine }\end{array}$ \\
\hline Ratio des biomasses fraiches & $\mathrm{Rbf}$ & $\begin{array}{l}\text { Quotient de la biomasse fraiche aérienne sur la } \\
\text { biomasse fraiche racinaire }\end{array}$ \\
\hline Ratio des biomasses sèches & Rbs & $\begin{array}{l}\text { Quotient de la biomasse sèche aérienne sur la } \\
\text { biomasse sèche racinaire }\end{array}$ \\
\hline
\end{tabular}

\section{RESULTATS}

\section{Effet du déficit hydrique sur le système aérien (tige et feuilles) des vitroplants}

La Figure 3 montre la réduction des paramètres morphologiques chez les deux morphotypes de voandzou après 30 jours de culture in vitro. La taille de la plantule et le nombre de feuilles diminuent avec l'augmentation de la concentration en agar du milieu. La réduction des paramètres aérien a été significative à partir de $18 \mathrm{~g} / \mathrm{L}$ d'agar, chez les morphotypes $\mathrm{Ci} 2$ et $\mathrm{Ci} 4$ (Tableau 2). L'analyse de variance réalisée a montré l'existence d'un effet concentration d'agar et d'un effet morphotype pour tous les paramètres de croissance du système aérien. De même, l'interaction (concentration d'agar $\mathrm{x}$ morphotype) a été significative au seuil de 5\%. Le déficit hydrique induit par l'agar a entrainé une diminution significative de tous les paramètres du système aérien. Cette réduction des paramètres de croissance a été observée à des concentrations qui différent d'un morphotype à un autre (Tableau 2). Les taux de réduction calculés à partir des valeurs des paramètres mesurés aux concentrations $6 \mathrm{~g} / \mathrm{l}$ (témoin) et $36 \mathrm{~g} / \mathrm{l}$ montrent que la hauteur de la plantule (Hpl), le nombre de feuilles (Nbf), la surface foliaire (SF), la biomasse fraiche aérienne (Bfa) et la biomasse sèche aérienne (Bsa) ont été respectivement de 39,46\%, $16,66 \%, 72,88 \%, 29,39 \%, 32,50 \%$ pour le morphotype Ci2 et $45,94 \%, 39,40 \%, 72,15 \%$, $53,80 \%, 39,18 \%$ pour le morphotype Ci4.

Le contenu relatif en eau des feuilles (CRE) a été influencé par l'augmentation de la concentration d'agar dans le milieu de croissance $(\mathrm{P}<0,001)$. Avec l'écotype Ci2, la réduction de $\mathrm{CRE}$ a été significative à partir de $12 \mathrm{~g} / \mathrm{l}$ d'agar dans le milieu de croissance alors que chez le morphotype Ci4, la diminution significative de CRE a été observée à partir de $24 \mathrm{~g} / \mathrm{l}$ d'agar (Figure 4). Les deux morphotypes ont présenté un comportement similaire en termes de contenu relatif en eau face au déficit hydrique (moins de 50\% de réduction). 


\section{Effet du déficit hydrique sur le système racinaire}

L'augmentation de la concentration d'agar dans le milieu de culture a entraîné une réduction significative de tous les paramètres $\mathrm{du}$ système souterrain des plantules des morphotypes $\mathrm{Ci} 2$ et $\mathrm{Ci} 4$ (Tableau 3). La réduction de la longueur de la racine principale (Lrp), des biomasses fraiche (Bfr) et sèche (Bsr) racinaire chez les morphotypes $\mathrm{Ci} 2$ et $\mathrm{Ci} 4$ est intervenue respectivement à partir de 12 et $18 \mathrm{~g} / \mathrm{l}, 12$ et $24 \mathrm{~g} / \mathrm{l}, 18$ et $30 \mathrm{~g} / \mathrm{l}$. Les taux de réduction pour la Lrp, le nombre de racines secondaires (Nrs), la Bfr et la Bsr ont été respectivement de $38,14 \%, 53,83 \%, 44,74 \%$, $52,25 \%$ pour le morphotype Ci2 et $58,23 \%$, $52,40 \%, 47,21 \%, 67,93 \%$ pour le morphotype Ci4. Ainsi, le morphotype Ci4 a été plus marqué par la réduction de croissance du système racinaire.

\section{Effet du déficit hydrique sur les ratios} système aérien / système racinaire

Les différents ratios ont été supérieurs ou égal à 1 (Tableau 4) pour toutes les concentrations d'agar. Le déficit hydrique occasionné par les concentrations croissantes d'agar a entrainé l'augmentation des ratios par rapport au témoin $(6 \mathrm{~g} / \mathrm{l})$ chez les deux morphotypes. Toutefois, pour chaque ratio, il n'existe pas de différence significative entre les morphotypes. Les différences ont été plutôt observées entre les concentrations d'agar. Ces résultats indiquent que le système racinaire a été plus affecté par le déficit hydrique que le système aérien chez les deux morphotypes.
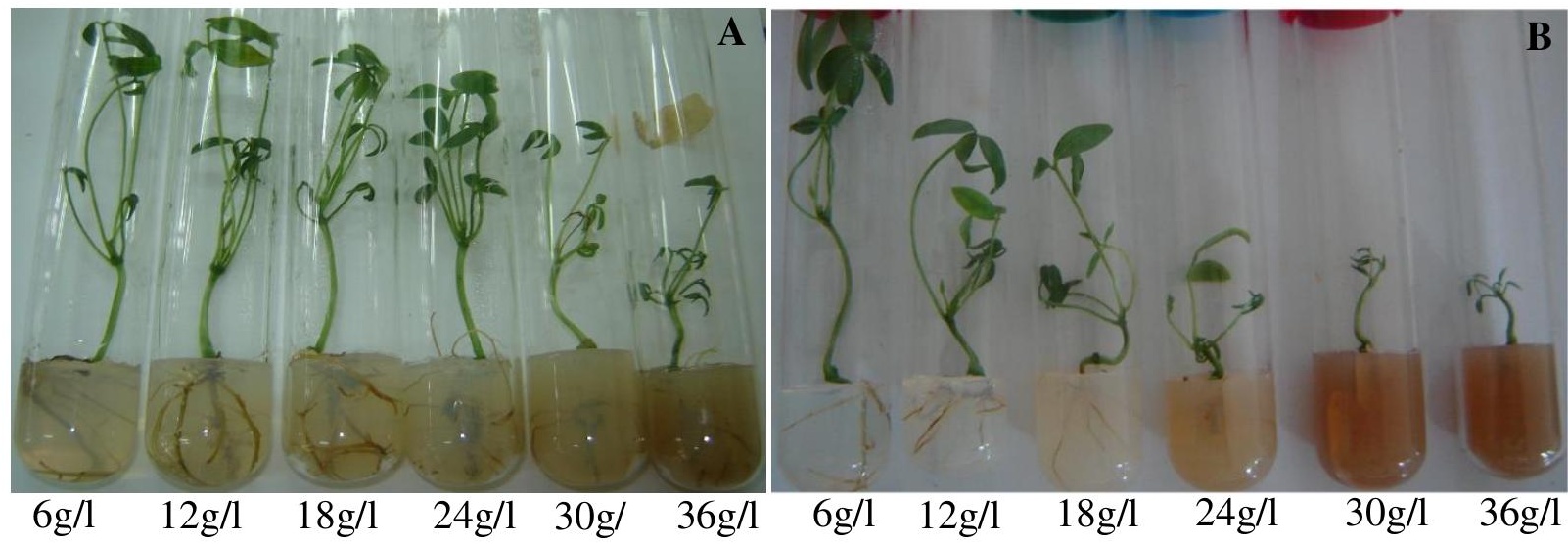

Figure 3: Plantules des morphotypes de voandzou soumises au déficit hydrique induit par l'agaragar. A : Plantules du morphotype Ci2 ; B : Plantules du morphotype Ci4.

Tableau 2 : Moyennes des paramètres de croissance du système aérien des plantules des morphotypes $\mathrm{Ci} 2$ et $\mathrm{Ci} 4$ soumis au déficit hydrique induit par l'agar-agar.

\begin{tabular}{|c|c|c|c|c|c|c|c|c|}
\hline \multirow{2}{*}{ Paramètres } & \multirow{2}{*}{ Morp } & \multicolumn{6}{|c|}{ Concentrations d'agar (g/l) } & \multirow[t]{2}{*}{$P$} \\
\hline & & 6 & 12 & 18 & 24 & 30 & 36 & \\
\hline \multirow{2}{*}{$\mathrm{Hpl}(\mathrm{cm})$} & $\mathrm{Ci} 2$ & $8,16^{\mathrm{a}}$ & $7,8^{\mathrm{a}}$ & $7,06^{\mathrm{b}}$ & $6,90^{\mathrm{b}}$ & $5,96^{\mathrm{c}}$ & $4,94^{\mathrm{d}}$ & \multirow{2}{*}{$<0,001$} \\
\hline & $\mathrm{Ci} 4$ & $6,26^{\mathrm{bc}}$ & $5,83^{\mathrm{c}}$ & $5,33^{\mathrm{cd}}$ & $3,33^{\mathrm{e}}$ & $3,34^{\mathrm{e}}$ & $2,93^{\mathrm{e}}$ & \\
\hline \multirow{2}{*}{$\mathrm{Nbf}$} & $\mathrm{Ci} 2$ & $5,40^{\mathrm{a}}$ & $4,96^{\mathrm{ab}}$ & $4,86^{\mathrm{ab}}$ & $5,066^{\mathrm{ab}}$ & $4,43^{b}$ & $4,5^{\mathrm{b}}$ & \multirow{2}{*}{$<0,001$} \\
\hline & $\mathrm{Ci} 4$ & $5,0^{\mathrm{ab}}$ & $4,46^{\mathrm{ab}}$ & $4,73^{\mathrm{ab}}$ & $3,90^{c}$ & $3,36^{\mathrm{d}}$ & $3,03^{\mathrm{d}}$ & \\
\hline \multirow{2}{*}{$\mathrm{Sf}\left(\mathrm{cm}^{2}\right)$} & $\mathrm{Ci} 2$ & $0,59^{\mathrm{ab}}$ & $0,34^{\text {cd }}$ & $0,27^{\mathrm{cd}}$ & $0,26^{\mathrm{cd}}$ & $0,18^{\mathrm{d}}$ & $0,16^{\mathrm{d}}$ & \multirow{2}{*}{$<0,001$} \\
\hline & $\mathrm{Ci} 4$ & $0,79^{\mathrm{a}}$ & $0,72^{\mathrm{a}}$ & $0,61^{\mathrm{ab}}$ & $0,45^{\mathrm{bc}}$ & $0,31^{\mathrm{cd}}$ & $0,22^{\mathrm{cd}}$ & \\
\hline
\end{tabular}




\begin{tabular}{llccccccc}
\hline \multirow{2}{*}{ Bfa (mg) } & Ci2 & $188,73^{\text {a }}$ & $171,24^{\text {ab }}$ & $156,71^{\text {ab }}$ & $150,44^{\mathrm{b}}$ & $137,64^{\mathrm{b}}$ & $133.26^{\mathrm{b}}$ & \multirow{2}{*}{${ }^{\mathrm{a}}$} \\
& Ci4 & $159,02^{\mathrm{ab}}$ & $146,45^{\mathrm{b}}$ & $138,23^{\mathrm{b}}$ & $75,73^{\mathrm{c}}$ & $72,46^{\mathrm{c}}$ & $80,93^{\mathrm{c}}$ & \\
\hline \multirow{2}{*}{ Bsa (mg) } & Ci2 & $21,78^{\mathrm{a}}$ & $17,93^{\mathrm{ab}}$ & $17,94^{\mathrm{ab}}$ & $16,55^{\text {bcd }}$ & $15,28^{\text {bcd }}$ & $14,70^{\text {bcd }}$ & \\
& Ci4 & $17,15^{\text {abc }}$ & $14,56^{\text {bcd }}$ & $14,06^{\text {bcd }}$ & $14,30^{\text {bcd }}$ & $11,10^{\mathrm{d}}$ & $10,43^{\mathrm{d}}$ & $<0,001$ \\
\hline
\end{tabular}

Pour chaque paramètre, les moyennes suivies d'une même lettre sont statistiquement identiques au seuil de 0,05 (Test de Newman-keuls). Morp : morphotype ; Hpl: Hauteur de la plantule; Nbf: Nombre de feuille; Sf: Surface foliaire; Bfa: Biomasse fraiche aérienne; Bsa: Biomasse sèche aérienne.

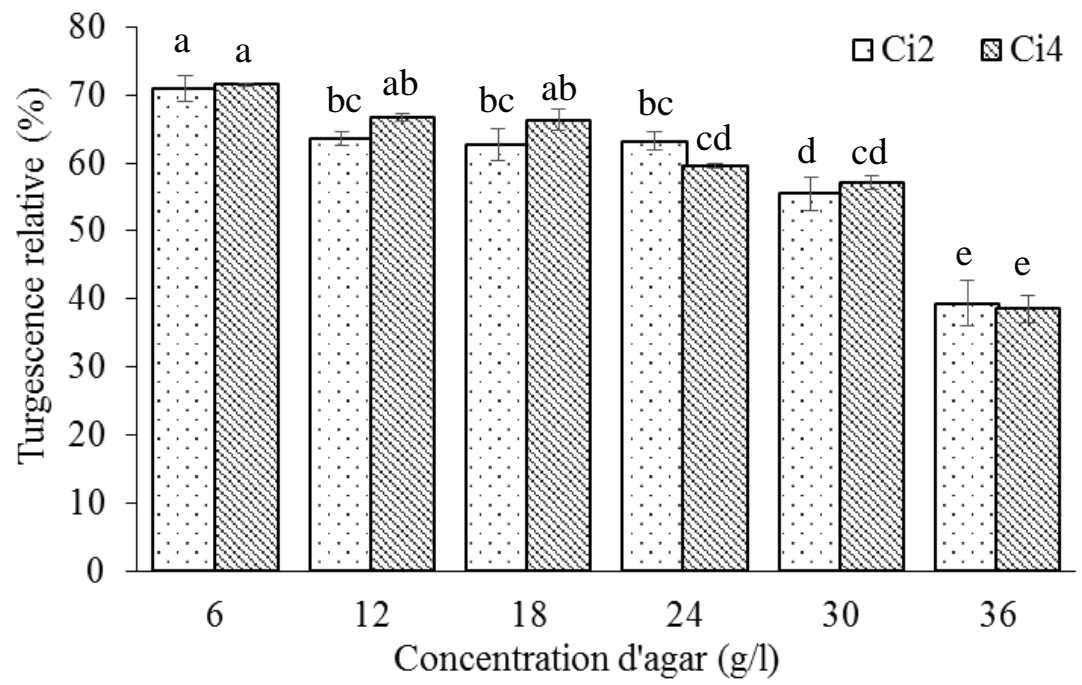

Figure 4 : Contenu relatif en eau des feuilles de deux morphotypes de voandzou soumis au déficit hydrique.

Les barres surmontées d'une même lettre montrent qu'il n'existe pas de différence entre les morphotypes au seuil de 0,05.

Tableau 3 : Moyennes des paramètres de croissance du système souterrain des plantules des morphotypes $\mathrm{Ci} 2$ et $\mathrm{Ci} 4$ soumis au déficit hydrique.

\begin{tabular}{|c|c|c|c|c|c|c|c|c|}
\hline \multirow{2}{*}{ Paramètres } & \multirow{2}{*}{ Morp } & \multicolumn{6}{|c|}{ Concentrations d'agar (g/l) } & \multirow{2}{*}{$P$} \\
\hline & & 6 & 12 & 18 & 24 & 30 & 36 & \\
\hline \multirow[b]{2}{*}{$\operatorname{Lrp}(\mathrm{cm})$} & $\mathrm{Ci} 2$ & $5,40^{\mathrm{a}}$ & $5,27^{\mathrm{a}}$ & $4,28^{\mathrm{b}}$ & $3,64^{\mathrm{bcd}}$ & $3,29^{\mathrm{cd}}$ & $3,34^{\text {cd }}$ & \multirow[b]{2}{*}{$<0,001$} \\
\hline & $\mathrm{Ci} 4$ & $4,86^{\mathrm{a}}$ & $3,96^{\mathrm{bc}}$ & $3,70^{\text {bcd }}$ & $2,96^{\mathrm{d}}$ & $2,26^{\mathrm{e}}$ & $2,03^{\mathrm{e}}$ & \\
\hline \multirow[b]{2}{*}{ Nrs } & $\mathrm{Ci} 2$ & $10,83^{\mathrm{a}}$ & $9,86^{\mathrm{ab}}$ & $7,50^{\mathrm{cd}}$ & $7,70^{\mathrm{cd}}$ & $5,205^{\mathrm{e}}$ & $5,00^{\mathrm{e}}$ & \multirow[b]{2}{*}{$<0,001$} \\
\hline & $\mathrm{Ci} 4$ & $9,16^{\mathrm{abc}}$ & $7,83^{\mathrm{cd}}$ & $6,26^{\mathrm{de}}$ & $4,56^{\mathrm{e}}$ & $4,76^{\mathrm{e}}$ & $4,36^{\mathrm{e}}$ & \\
\hline \multirow{2}{*}{ Bfr (mg) } & $\mathrm{Ci} 2$ & $157,02^{\mathrm{a}}$ & $122,98^{\mathrm{b}}$ & $96,37^{\mathrm{c}}$ & $98,65^{\mathrm{c}}$ & $86,58^{c}$ & $86,76^{\mathrm{c}}$ & \multirow{2}{*}{$<0,001$} \\
\hline & $\mathrm{Ci} 4$ & $84,31^{\mathrm{c}}$ & $71,88^{\mathrm{cd}}$ & $76,33^{\mathrm{cd}}$ & $52,20^{\mathrm{de}}$ & $49,60^{\mathrm{de}}$ & $44,50^{\mathrm{e}}$ & \\
\hline \multirow{2}{*}{ Bsr (mg) } & $\mathrm{Ci} 2$ & $22,39^{\mathrm{a}}$ & $19,66^{\mathrm{ab}}$ & $16,75^{\mathrm{bc}}$ & $14,69^{\mathrm{cd}}$ & $12,19^{\mathrm{de}}$ & $10,69^{\text {def }}$ & \multirow{2}{*}{$<0,001$} \\
\hline & $\mathrm{Ci} 4$ & $13,97^{\text {cde }}$ & $12,24^{\mathrm{def}}$ & $11,40^{\text {def }}$ & $9,76^{\mathrm{ef}}$ & $7,93^{\mathrm{f}}$ & $4,48^{g}$ & \\
\hline
\end{tabular}

Pour chaque paramètre, les moyennes suivies d'une même lettre sont statistiquement identiques au seuil de 0,05 (Test de Newman-keuls). Lrp: Longueur de la racine principale; Nrs: Nombre de racines secondaires; Bfr: Biomasse fraiche racinaire; Bsr: Biomasse sèche racinaire. 
Tableau 4 : Moyennes des ratios des paramètres de croissance des systèmes aérien et souterrain des plantules des morphotypes $\mathrm{Ci} 2$ et $\mathrm{Ci} 4$ soumis au déficit hydrique.

\begin{tabular}{|c|c|c|c|c|c|c|c|c|}
\hline \multirow{2}{*}{ Paramètres } & \multirow{2}{*}{ Morp } & \multicolumn{6}{|c|}{ Concentrations d'agar (g/l) } & \multirow{2}{*}{$\frac{\text { Statistiques }}{P}$} \\
\hline & & 6 & 12 & 18 & 24 & 30 & 36 & \\
\hline \multirow{2}{*}{ Rhl } & $\mathrm{Ci} 2$ & $1,51^{\mathrm{cd}}$ & $1,47^{\mathrm{cd}}$ & $1,65^{\mathrm{abc}}$ & $1,89^{\mathrm{a}}$ & $1,82^{\mathrm{ab}}$ & $1,47^{\mathrm{bcd}}$ & \multirow{2}{*}{$<0,001$} \\
\hline & $\mathrm{Ci} 4$ & $1,29^{\mathrm{cd}}$ & $1,47^{\mathrm{bcd}}$ & $1,44^{\text {bcd }}$ & $1,47^{\text {bcd }}$ & $1,45^{\text {bcd }}$ & $1,15^{\mathrm{d}}$ & \\
\hline \multirow{2}{*}{ Rbf } & $\mathrm{Ci} 2$ & $1,20^{\mathrm{a}}$ & $1,39^{\mathrm{b}}$ & $1,62^{\mathrm{c}}$ & $1,58^{\mathrm{c}}$ & $1,59^{\mathrm{c}}$ & $1,56^{\mathrm{cd}}$ & \multirow{2}{*}{$<0,001$} \\
\hline & $\mathrm{Ci} 4$ & $1,83^{\mathrm{cd}}$ & $1,89^{\mathrm{d}}$ & $2,04^{\mathrm{e}}$ & $1,61^{\mathrm{c}}$ & $1,46^{\mathrm{bc}}$ & $1,78^{\mathrm{cd}}$ & \\
\hline \multirow{2}{*}{ Rbs } & $\mathrm{Ci} 2$ & $0,97^{\mathrm{b}}$ & $0,91^{\mathrm{b}}$ & $1,01^{\mathrm{b}}$ & $1,23^{\mathrm{b}}$ & $1,26^{\mathrm{b}}$ & $1,37^{\mathrm{b}}$ & \multirow{2}{*}{$<0,001$} \\
\hline & $\mathrm{Ci} 4$ & $1,22^{\mathrm{b}}$ & $1,18^{\mathrm{b}}$ & $1,25^{\mathrm{b}}$ & $1,48^{\mathrm{b}}$ & $1,56^{\mathrm{b}}$ & $2,16^{\mathrm{a}}$ & \\
\hline
\end{tabular}

Pour chaque paramètre, les moyennes suivies d'une même lettre sont statistiquement identiques au seuil de 0,05 (Test de Newman-keuls). Rhl: ratio hauteur de la plante et longueur de la racine principale; Rbf: Ratio des biomasses fraiches; Rbs: Ratio des biomasses sèches.

\section{DISCUSSION}

L'évaluation de l'aptitude à la résistance à la sècheresse en conditions de culture in vitro de deux morphotypes de voandzou a montré que le déficit hydrique induit par l'agar réduit significativement la hauteur de la plantule $(\mathrm{Hpl})$, le nombre de feuilles (Nbf) et la surface foliaire (Sf) des plantules. Ces résultats ont été aussi obtenus sous serre par Imorou et al. (2018) chez le maïs. $\mathrm{La}$ réduction de la $\mathrm{Hpl}$ est la conséquence d'une croissance cellulaire limitée qui probablement est due à la diminution de l'absorption de l'eau et des substances nutritives par les plantules. Selon Mwale et al. (2007), la réduction du Nbf par plantule pourrait être l'un des mécanismes élaborés par les plantes de voandzou pour réduire la surface foliaire totale en condition de déficit hydrique. Par ailleurs, la diminution de la SF permet à la plante de réduire la perte en eau afin de résister au déficit hydrique. La plante ajuste sa taille à l'eau disponible dans le milieu en réduisant la surface et/ou le nombre de ses feuilles (Hireche, 2006).

La baisse significative des biomasses fraiches aériennes et souterraines des plantules chez les deux morphotypes par rapport au témoin a été aussi observée chez Abelmoschus esculentus (Aziafekey et al., 2019) et Macrotyloma geocarpum (Nguinambaye et al., 2020). Cette baisse serait due à la réduction de l'expansion et de la division cellulaire. En effet, la diminution de l'entrée d'eau dans les cellules affecte tout le métabolisme cellulaire qui, par conséquent, provoquerait un ralentissement de la croissance de la plantule. Selon Mwale et al. (2007), l'influence du déficit hydrique sur le voandzou concorde avec le comportement général de fermeture des stomates afin de réduire les pertes en eau. Cette fermeture entraine également une réduction de la diffusion du $\mathrm{CO}_{2}$ lors de la photosynthèse et donc une baisse significative de la production de matière (Xu et al., 2010). La biomasse aérienne a été moins affectée par le stress hydrique. Il a donc eu une allocation préférentielle de la matière sèche vers la partie aérienne (tige plus feuilles). Contrairement au cas du voandzou, les travaux réalisés par Turk et al. (2004) et Santos et al. (2020) ont montré que la croissance racinaire a été moins affectée que celle de la tige chez la lentille et le niébé.

La réduction significative de la longueur de la racine notée en présence de concentrations croissantes d'agar a été aussi observée en conditions in vitro chez le niébé (Badiane et al., 2004) et chez la pomme de terre (Gopal et al., 2008). Contrairement à ces observations, Mohammed et al. (2003) ont noté une augmentation de la longueur de la racine chez l'argentier en condition de déficit hydrique, ce qui serait ainsi un mécanisme d'adaptation développé par cette plante. 
Les folioles des deux morphotypes ont généralement présenté des contenus relatifs en eau (CRE) supérieurs à 50\%. Le maintien de valeurs relativement élevées de CRE malgré le déficit hydrique apparaît comme un trait de résistance à la sécheresse des morphotypes de voandzou étudiés.

Un effet morphotype a aussi été observé chez le voandzou. La HP, la Lap, le Nbf, le PFRa et le PSSa peuvent être considérés comme discriminants et donc être utilisés pour cribler les morphotypes de voandzou en vue de sélectionner ceux qui sont tolérants à la sécheresse. Le morphotype Ci2 serait plus tolérant au déficit hydrique comparativement au morphotype Ci4. Evidemment, la différence de comportement ainsi exprimée résulterait d'un effet génotype. L'aptitude du morphotype Ci2 à tolérer le stress hydrique par rapport au morphotype Ci4 justifie probablement sa large adoption par les paysans du district des savanes (principale aire de production) de la Côte d'Ivoire (Touré et al., 2013).

\section{Conclusion}

Les expérimentations conduites en conditions contrôlées, ont montré que le déficit hydrique induit par les différentes concentrations d'agar-agar affecte significativement la croissance des plantules in vitro chez le voandzou. La stratégie développée par les morphotypes en situation de disponibilité réduite en eau a généralement consisté en une réduction significative des paramètres de croissance évalués. Toutefois, l'effet du déficit hydrique sur la croissance a varié selon les organes et le morphotype utilisé. Le système racinaire des plantules a été plus sensible au déficit hydrique que le système aérien. Les paramètres de croissance ont significativement été plus réduits chez le morphotype Ci4. Ce morphotype a donc été plus affecté par le déficit hydrique et par conséquent il est considéré comme étant le moins tolérant au manque d'eau. Les futurs travaux seront élargis à un nombre plus élevé de morphotypes et prendraient en compte les marqueurs biochimiques et moléculaires de la résistance au déficit hydrique chez le voandzou. Par ailleurs, la technique développée pourrait favoriser la sélection de variétés tolérantes dans les régions arides et semi-arides où la disponibilité en eau demeure le principal facteur limitant la productivité des espèces végétales.

\section{CONFLIT D'INTERETS}

Les auteurs de cet article déclarent qu'il n'y a aucun conflit d'intérêts pour cette publication.

\section{CONTRIBUTIONS DES AUTEURS}

YT et MK ont conçu le projet. YT et TK ont contribué à l'analyse statistique. LFC et NS ont participé à la recherche bibliographique. Le projet a été supervisé par MK. Chacun de ces auteurs a participé à la rédaction du manuscrit.

\section{REFERENCES}

Aziafekey M, Odah K, Atayi A. 2019. Effet de différentes modalités de stress hydrique sur la production et la teneur en éléments minéraux du gombo [Abelmoschus esculentus (L.) Moench]. Int. J. Biol. Chem. Sci., 7(4): 1515-1523. DOI: 10.4314/ijbcs.v7i4.8

Badiane FA, Diouf D, Sané D, Diouf O, Goudiaby V, Diallo N. 2004. Screening cowpea [Vigna unguiculata (L.) Walp.] Varieties by inducing water deficit and RAPD analyses. Afr. J. Biotechnol., 3(3): 174-178. DOI: $10.5897 / A J B 2004.000-$ 2030

Basu S, Mayes S, Davey M, Jeremy A, AzamAli SN, Mithen R, Pasquet RS. 2007. Inheritance of domestication traits in Bambara groundnut (Vigna subterranea (L.) Verdc). Euphytica, 157: 59-68. DOI: 10.1007/s10681-007-9396-4

Boutraa T, Sanders FE. 2001. Influence of Water Stress on Grain Yield and Vegetative Growth of Two Cultivars of Bean (Phaseolus vulgaris L.). J. Agro. Crop Sci., 187: 251-257. DOI: 10.1046/j.1439-037X.2001.00525.x

Gopal J, Iwama K, Jitsuyama Y. 2008. Effect of water stress mediated through agar on in vitro growth of potato. In Vitro Cell. 
Dev. Biol. Plant, 44: 221-228. DOI: 10.1007/s11627-007-9102-1

Hireche Y. 2006. Réponse de la luzerne (Medicago sativa $\mathrm{L}$ ) au stress hydrique et à la profondeur de semis. Mémoire de magistère en Science agronomique, Département d'Agronomie, Université Al Hadj Lakhdar-Batna, Algérie, 83 p

Imorou L, Ahoton EL, Zoumarou-Wallis N, Kanlindogbe C. 2018. Water stress effect on agro-morphological and physiological parameters of three local cultivars of maize (Zea mays L.) of South Benin. Int. J. Biol. Chem. Sci., 12(5): 2294-2308. DOI: $10.4314 /$ ijbcs.v12i5.29

Jensen CR, Jacobsen S, Andersen MN, Nūñez $\mathrm{N}$, Andersen SD, Rasmussen L, Mogensen VO. 2000. Leaf gas exchange and water relation characteristics of field quinoa (Chenopodium quinoa Willd.) during soil drying. Eur. J. Agron., 13: 1125. DOI: $\quad 10.1016 / \mathrm{S} 1161-$ 0301(00)00055-1

Lavorel S. 2013. Plant functional effects on ecosystem services. Journal of Ecology, 101: 4-8. DOI: 10.1111/13652745.12031

Mohammed RT, Brrechi A, Haloui B. 2003. Effet du polyéthylène glycol sur la germination et la croissance in vitro de l'arganier (Argania spinosa L. Skeels). Sécheresse, 14(1): 7-23.

Mwale SS, Azam-Ali SN, Massawe FJ. 2007. Growth and development of bambara groundnut (Vigna subterranea) in response to soil moisture. Dry matter and yield. Eur. J. Agron., 26(4): 345-353. DOI: $10.1016 /$ j.eja. 200609.007

Nguinambaye MM, Nana R, Djinet IA, Tamini Z. 2020. Quelques paramètres physiologiques et constituants biochimiques des organes de la lentille de terre (Macrotyloma geocarpum) en conditions de stress hydrique. Int. J. Biol. Chem. Sci., 14(4): 1228-1240. DOI: 10.4314/ijbcs.v14i4.6
Onwugbuta-Enyi J. 2004. Water balance and proximate composition in cowpea (Vigna unguiculata (L.) Walps) seedlings exposed to drought and flooding stress. $J$. Appl. Sci. Environ. Manage., 8(2): 55-57. DOI: 10.4314 /jasem.v8i2.17241

Ouoba A, Ouedraogo M, Sawadogo M, Nadembega S. 2016. Aperçu de la culture du voandzou (Vigna subterranea (L.) Verdcourt) au Burkina Faso: enjeux et perspectives d'amélioration de sa productivité. Int. J. Biol. Chem. Sci., 10(2): $\quad$ 652-665. DOI: http://dx.doi.org/10.4314/ijbcs.v10i2.17

Santos R, Carvalho M, Eduardo R, Carnide V, Castro I. 2020. Root and AgroMorphological Traits Performance in Cowpea under Drought Stress. Agronomy, 10: 1-20. DOI: 10.3390/agronomy 10101604

Touré Y, Koné M, Kouakou HT, Koné D. 2012. Agromorphological and Phenological Variability of 10 Bambara Groundnut [Vigna subterranea (L.) Verdc. (Fabaceae)] landraces cultivated in the Ivory Coast. Tropicultura, 30(4): 216-221.

Touré Y, Koné M, Silué S, Kouadio YJ. 2013. Prospection, collecte et caractérisation agromorphologique des morphotypes de voandzou [Vigna subterranea (L.) Verdc. (Fabaceae)] de la zone savanicole en Côte d'Ivoire. Eur. Sci. J., 9(24): 308-325.

Turk MA, Tawaha AM, Lee KD. 2004. Seed germination and seedling growth of three lentil cultivars under moisture stress. Asian J. Plant Sci., 3(3): 394-397. DOI: 10.3923/ajps.2004.394.397

Xu Z, Zhou G, Shimizu H. 2010. Plant responses to drought and rewatering. Plant Signal. Behav., 5: 649-654. DOI: 10.4161/psb.5.6.11398 